\title{
Effect of the Polydopamine Composite Method on Structural Coloration: Comparison of Binary and Unary Assembly of Colloidal Particles
}

Takeshi Iwasaki ${ }^{\dagger} \star \star$, Shotaro Harada ${ }^{\dagger}$, Taku Okoshi ${ }^{\dagger}$, Miyu Moriya ${ }^{\dagger}$, Takashi Kojima ${ }^{\dagger}$, Keiki Kishikawa ${ }^{\dagger}$, and Michinari Kohri*†

$\dashv$ Department of Applied Chemistry and Biotechnology, Graduate School of Engineering, Chiba University, 1-33 Yayoi-cho, Inage-ku, Chiba 263-8522, Japan

Banknote Department, Head Office, National Printing Bureau, 2-2-5 Toranomon, Minato-ku, Tokyo 105-8445, Japan

Corresponding Author

*E-mail address: kohri@faculty.chiba-u.jp (M. K.) 


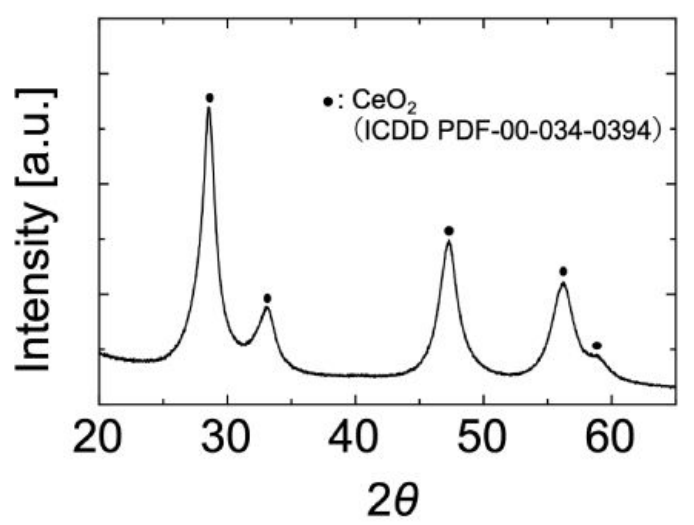

Figure S1. XRD pattern of the PVP-coated $\mathrm{CeO}_{2}$ particles.

(a)

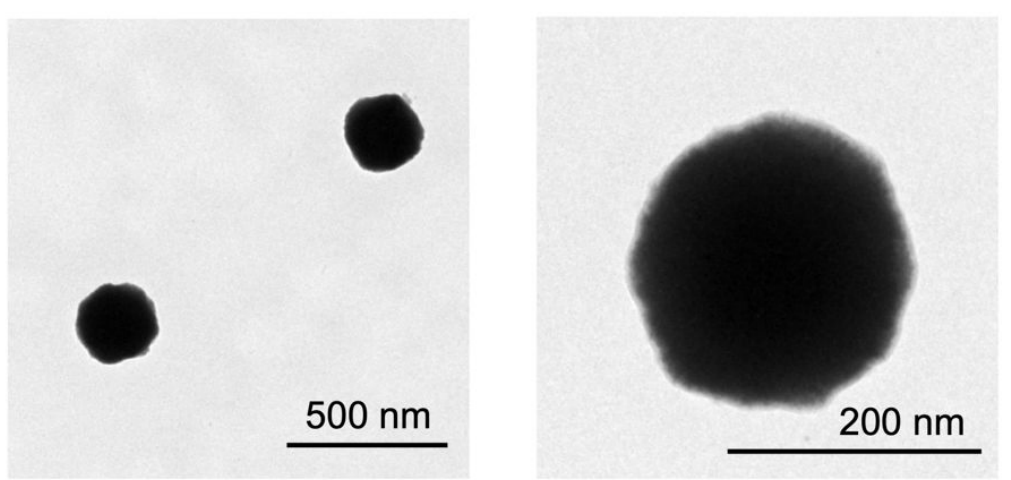

(b)

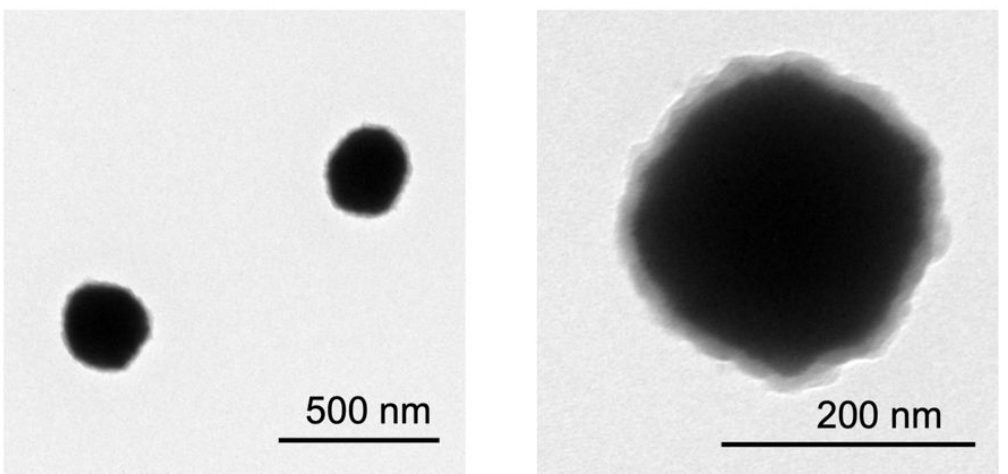

Figure S2. TEM images of (a) $\mathrm{CeO}_{2}$ core particles and (b) $\mathrm{CeO}_{2} @ \mathrm{PDA}$ core-shell particles. 


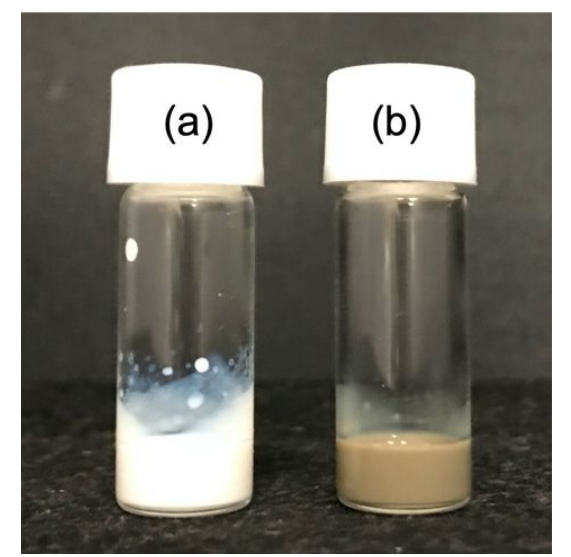

Figure S3. Photographs of the water dispersion of (a) $\mathrm{CeO}_{2}$ core particles and (b) $\mathrm{CeO}_{2} @$ PDA core-shell particles.

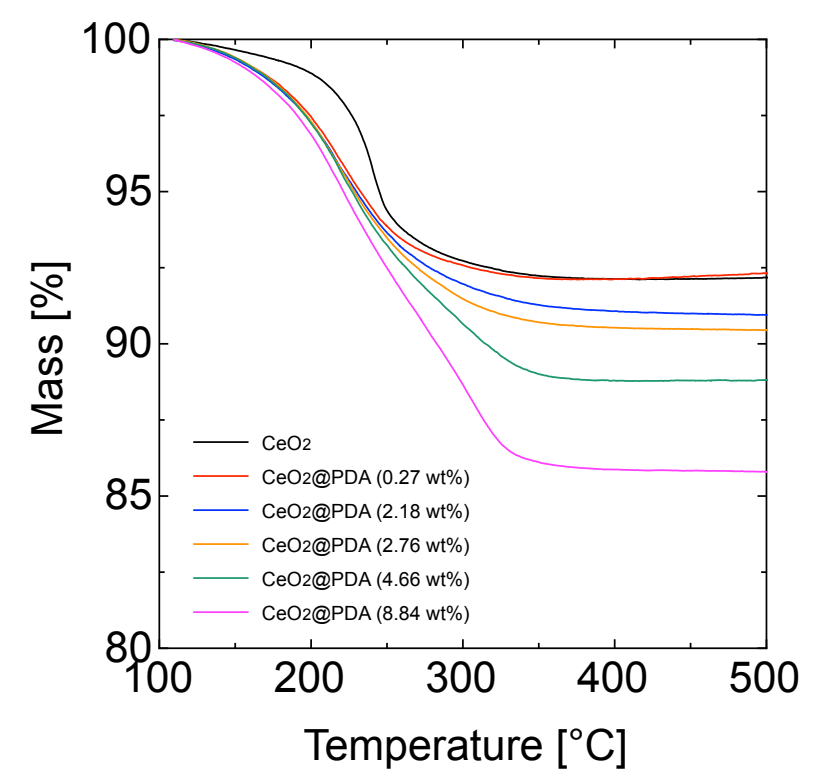

Figure S4. TG analysis results of $\mathrm{CeO}_{2}$ core particles and $\mathrm{CeO}_{2} @ \mathrm{PDA}$ core-shell particles. 
(a) PDA amount [wt\%] $2.182 .83 \quad 4.65 \quad 8.93$
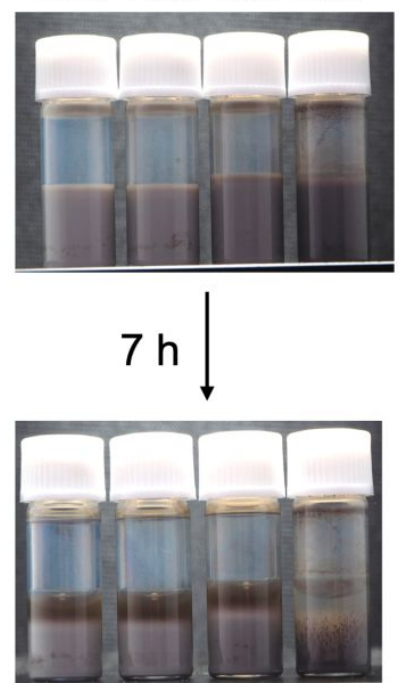

(b) PDA amount [wt\%] $2.18 \quad 2.76 \quad 4.66 \quad 8.84$
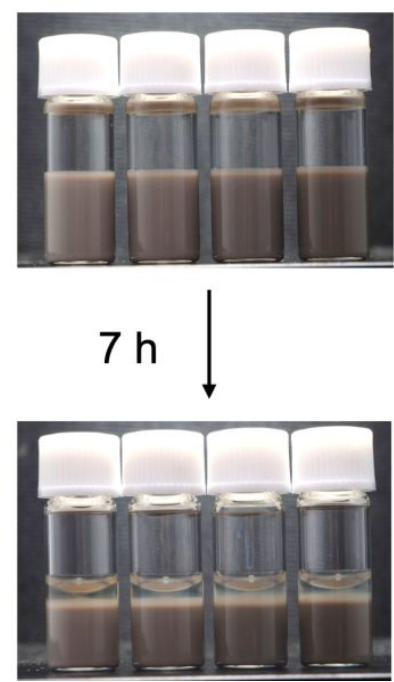

Figure S5. Photographs of aqueous dispersion ( $2 \mathrm{wt} \%$ ) of particles after 0 and $7 \mathrm{~h}$. (a) binary coassembly of $\mathrm{CeO}_{2}$ and PDA particles and (b) unary assembly of $\mathrm{CeO}_{2} @$ PDA core-shell particles.

(a) PDA amount [wt\%]

0.26

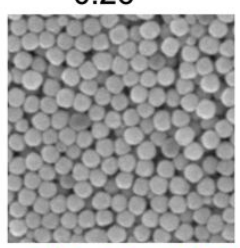

(b)

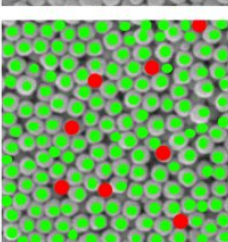

Percentage of PDA particles [\%]
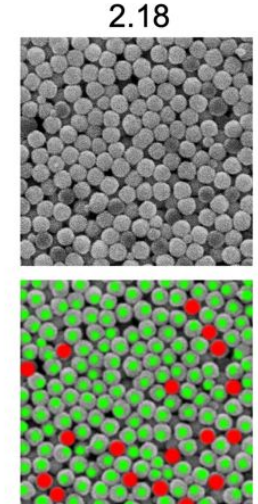

9.9
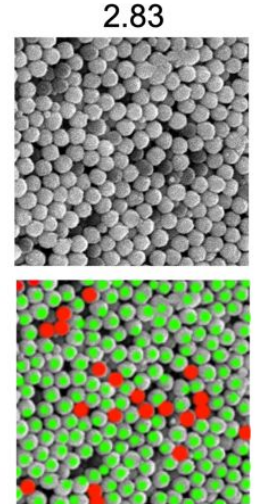

10.8

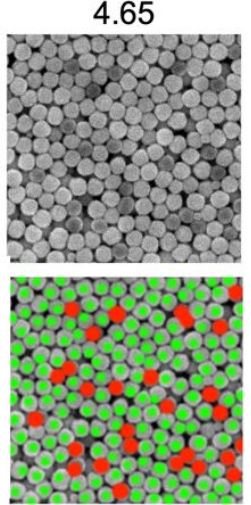

14.4
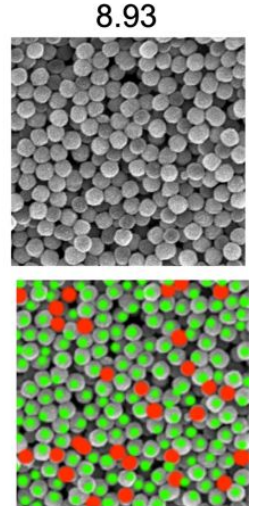

14.6

Figure S6. (a) SEM images of binary co-assembly of $\mathrm{CeO}_{2}$ and PDA particles and initial PDA amount. (b) SEM images of binary coassembly of $\mathrm{CeO}_{2}$ and PDA particles. The green and red circles in the SEM images of (b) indicate $\mathrm{CeO}_{2}$ and PDA particles, respectively. The number represents the percentage of PDA particles on the material surface. 
(a)

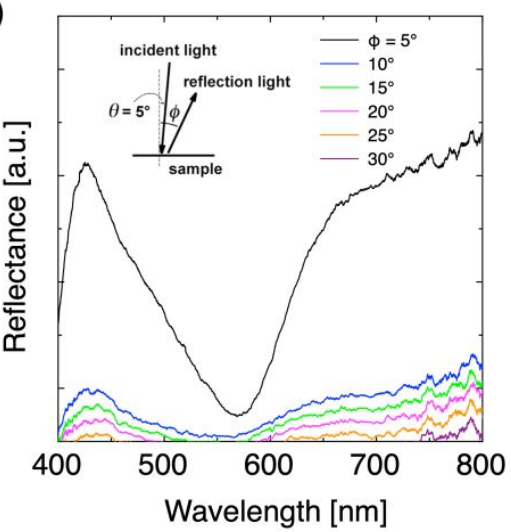

(b)

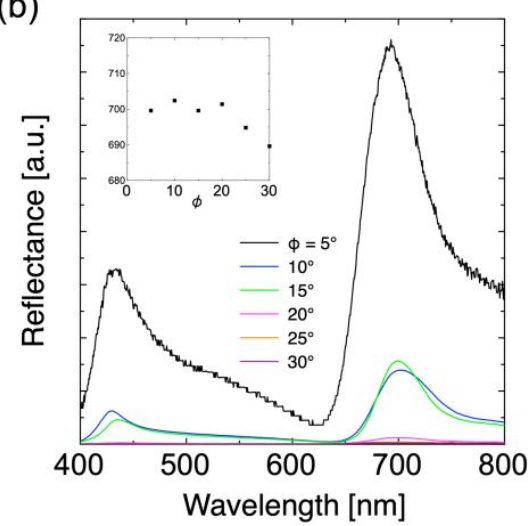

Figure S7. Reflection spectra from various angles of pellet samples made from (a) binary coassembly and (b) unary assembly. The inset in (b) shows the peak top position of the reflection spectrum of the pellet.

Table S1. The $a^{*}, b^{*}, C^{*}, L^{*}$, and $h$ values of pellet samples

\begin{tabular}{|c|c|c|c|c|c|c|}
\hline \multicolumn{2}{|l|}{$\begin{array}{l}\text { PDA amount of sample } \\
{[\mathrm{wt} \%]}\end{array}$} & $a^{*}$ & $b^{*}$ & $C^{*}$ & $L^{*}$ & $h$ \\
\hline Core $\mathrm{CeO}_{2}$ & 0 & 6.85 & -7.50 & 10.2 & 33.67 & 312 \\
\hline \multirow{4}{*}{$\begin{array}{c}\text { Binary } \\
\text { coassembly }\end{array}$} & 0.26 & 10.90 & -13.79 & 17.58 & 22.57 & 308 \\
\cline { 2 - 7 } & 2.18 & 18.24 & -20.73 & 27.61 & 18.19 & 311 \\
\cline { 2 - 7 } & 2.83 & 23.64 & -19.23 & 30.47 & 12.40 & 321 \\
\cline { 2 - 7 } & 4.65 & 21.13 & -20.80 & 29.65 & 13.36 & 316 \\
\cline { 2 - 7 } & 8.93 & 23.55 & -18.43 & 29.90 & 15.26 & 322 \\
\cline { 2 - 7 } & 0.27 & 30.23 & -12.29 & 32.63 & 25.69 & 338 \\
\cline { 2 - 7 } Unary & 2.18 & 28.95 & -16.65 & 33.40 & 25.22 & 330 \\
\cline { 2 - 7 } assembly & 2.76 & 28.67 & -15.76 & 32.72 & 27.32 & 331 \\
\cline { 2 - 7 } & 4.66 & 13.52 & -15.79 & 20.79 & 17.32 & 311 \\
\cline { 2 - 7 } & 8.84 & 6.00 & -17.38 & 18.4 & 16.45 & 289 \\
\hline
\end{tabular}

